\title{
Critical slowing down of relaxation dynamics near the Curie temperature in the relaxor $\mathrm{Pb}\left(\mathrm{Sc}_{0.5} \mathrm{Nb}_{0.5}\right) \mathrm{O}_{3}$
}

\author{
Muhtar Ahart, ${ }^{1, a)}$ Anwar Hushur, ${ }^{2, b}$ Yonghong Bing, ${ }^{3}$ Zuo-Guang Ye, ${ }^{3}$ Russell J. Hemley, ${ }^{1}$ \\ and Seiji Kojima ${ }^{2, c)}$ \\ ${ }^{1}$ Geophysical Laboratory, Carnegie Institution of Washington, Washington, DC 20015, USA \\ ${ }^{2}$ Institute for Materials Science, University of Tsukuba, Tsukuba, Ibaraki 305-8573, Japan \\ ${ }^{3}$ Department of Chemistry and $4 D$ Laboratories, Simon Fraser University, Burnaby, \\ British Columbia V5A 1S6, Canada
}

(Received 14 January 2009; accepted 16 March 2009; published online 8 April 2009)

\begin{abstract}
Brillouin spectroscopy of $\mathrm{Pb}\left(\mathrm{Sc}_{0.5} \mathrm{Nb}_{0.5}\right) \mathrm{O}_{3}$ single crystals reveals an order-disorder ferroelectric phase transition occurs at $110{ }^{\circ} \mathrm{C}$ upon cooling. A softening of the longitudinal acoustic (LA) mode is observed that can be attributed to the coupling between polar nanoregions and acoustic modes. A critical slowing down of the central peak, a feature of order-disorder ferroelectric phase transitions, is observed near $T_{c}$. The similarity in temperature dependences of the two kinds of relaxation times determined from the central peak and LA mode suggests that the changes in the central peak arise from local polarization fluctuations in the polar nanoregions. (C) 2009 American Institute of Physics. [DOI: $10.1063 / 1.3115765]$
\end{abstract}

Relaxor ferroelectrics exhibit a strong frequency dispersive dielectric susceptibility with very broad and smooth temperature dependences associated with the presence of small polar clusters-so-called polar nanoregions (PNRs). ${ }^{1}$ Their high permittivity, high piezoelectric properties, as well as other properties make them suitable for applications in the field of piezoelectric devices such as sonar or medical imaging. ${ }^{2}$ While extensive theoretical and experimental studies have advanced our understanding of relaxors, many physical properties of these materials still remain unclear. For example, because of the small size and random nature of PNRs, we still lack a clear understanding of their size distribution, thickness, and roughness of their boundaries, their dynamics, and how they affect ferroelectric properties.

This paper focuses on disordered $\mathrm{Pb}\left(\mathrm{Sc}_{1 / 2} \mathrm{Nb}_{1 / 2}\right) \mathrm{O}_{3}$ (PSN) materials with implications for perovskite relaxors such as $\mathrm{Pb}\left(\mathrm{Mg}_{1 / 3} \mathrm{Nb}_{2 / 3}\right) \mathrm{O}_{3}$ (PMN). Conventional dielectric spectroscopy gives spatial averages of properties of a sample as a whole and may obscure microscopic origins. Although Refs. 3-5 discussed fast relaxation in relaxors obtained using a combination of microwave and terahertz dielectric spectroscopic measurements, the spectral range from 8 to $90 \mathrm{GHz}$ is not covered within those techniques. Methods such as microBrillouin scattering spectroscopy, which can cover 1 to 100 $\mathrm{GHz}$, are needed to identify the microscopic origin of macroscopic behavior. The observations of strong first-order Raman scattering in cubic lead perovskite relaxors ${ }^{6}$ indicate that the potential energy of PNRs can be described by a multiwell structure in the high-temperature phase, ${ }^{7}$ with hopping between wells that causes high-frequency relaxation. Therefore, it is important to study the dynamical behavior of PNRs in the high-frequency range. Here we employed micro-Brillouin scattering to investigate the temperature dependences of acoustic modes and central peak (CP), in PSN from 0 to $600{ }^{\circ} \mathrm{C}$.

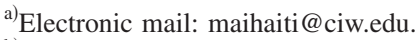

${ }^{b}$ Present address: Hawaii Institute of Geophysics and Planetology, University of Hawaii, Honolulu, Hawaii 96822, USA.

${ }^{c)}$ Electronic mail:kojima@ims.tsukuba.ac.jp.
}

PSN exhibits more complex behavior than PMN because of the possibility to vary its $B$-site chemical order. Although highly ordered PSN samples exhibit a normal paraelectric to ferroelectric transition on cooling, ${ }^{8}$ highly disordered samples first enter a relaxor state on cooling followed by a cubic relaxor to rhombohedral ferroelectric transition. ${ }^{9,10}$ This property of PSN has stimulated theoretical calculations ${ }^{11}$ because the ferroelectric ground state $(R 3 m)$ (Ref. 9) is known. The calculations on disordered PSN (hereafter $d$-PSN) suggest that relaxors can be considered as normal ferroelectrics existing in random fields. ${ }^{11}$

Single crystals of $\mathrm{Pb}\left(\mathrm{Sc}_{1 / 2} \mathrm{Nb}_{1 / 2}\right) \mathrm{O}_{3}$ used in this study were grown by a high-temperature solution method. X-ray diffraction confirmed the disordered perovskite structure. A $3+3$ pass tandem Fabry-Pérot interferometer was employed to investigate the temperature dependence of Brillouin shifts in a $d$-PSN single crystals in a backscattering geometry. ${ }^{12} \mathrm{~A}$ single frequency $\mathrm{Ar}^{+}$ion laser at $514.5 \mathrm{~nm}$ and power of 50 $\mathrm{mW}$ was used with a conventional photon-counting system and multichannel analyzer. A pseudocubic (001)-orientated $d$-PSN single crystal with a dimension of $1 \times 1 \times 0.5 \mathrm{~mm}^{3}$ was put in a cryostat cell (Linkam THMS 600) with temperature variation from -190 to $600{ }^{\circ} \mathrm{C}$ and stability $\pm 0.1^{\circ} \mathrm{C}$. The sample cell with $X-Y$ adjustment was mounted on an optical microscope (Olympus BH-2).

Selected Brillouin spectra are shown in Fig. 1. There are two pairs of peaks in each spectrum with a Rayleigh peak at zero frequency. One pair corresponds to the quasilongitudinal acoustic mode (LA mode), the other pair corresponds to the quasitransverse acoustic modes (TA mode), respectively. Notably TA modes are only visible in the low-temperature regions. The lack of TA modes in the high temperature cubic phase arises from the backscattering geometry; thus we will not discuss observed TA modes here. A broad relaxation mode (quasielastic scattering) around the Rayleigh peak is clearly observed (Fig. 1) with a marked temperature dependence. The acoustic modes were analyzed with a sum of damped harmonic oscillators, and the $\mathrm{CP}$ was analyzed with a Lorentzian function centered at zero frequency. The temperature dependences of linewidth and frequency of the LA 


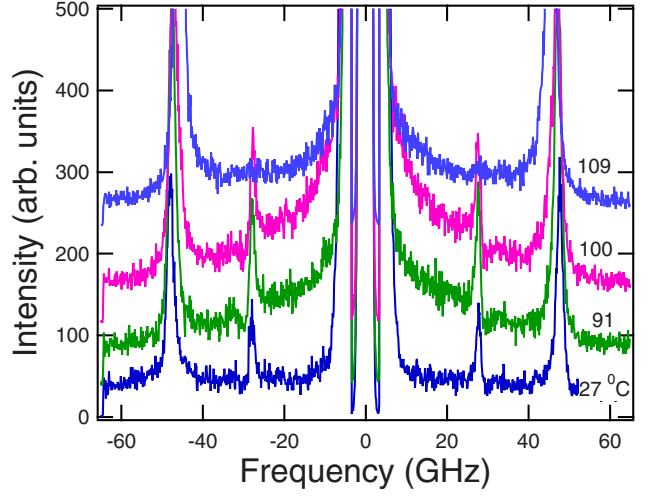

FIG. 1. (Color online) Representative Brillouin spectra at selected temperatures of $d$-PSN crystals. There are two pairs of peaks in each spectrum and an additional Rayleigh peak at zero frequency (elastic and quasielastic scattering). One pair corresponds to the quasi-LA mode, the other pair corresponds to the quasi-TA mode, respectively. A relaxation mode (quasielastic scattering) is clearly observed near the elastic Rayleigh peak.

mode clearly exhibit elastic anomalous near the transition point at $110{ }^{\circ} \mathrm{C}$ and significant softening of elastic mode between $T_{c}$ and $600{ }^{\circ} \mathrm{C}$ (Fig. 2).

Anomalous changes in elastic properties due to the ferroelectric phase transitions can be described by Landau theory, according to which the anomalous behavior at the Curie temperature $T_{c}$ arises from coupling of the strain and order parameter in a free-energy expansion. The coupling occurs because the acoustic wave changes the magnitude of ion displacements. If the dominant coupling term in the interacting potential is electrostrictive (a common assumption in the Pb-based relaxors), the Brillouin shift should be constant above $T_{c}$. With this coupling term in the free-energy expansion, the acoustic mode linewidth should show a $\lambda$-type peak.

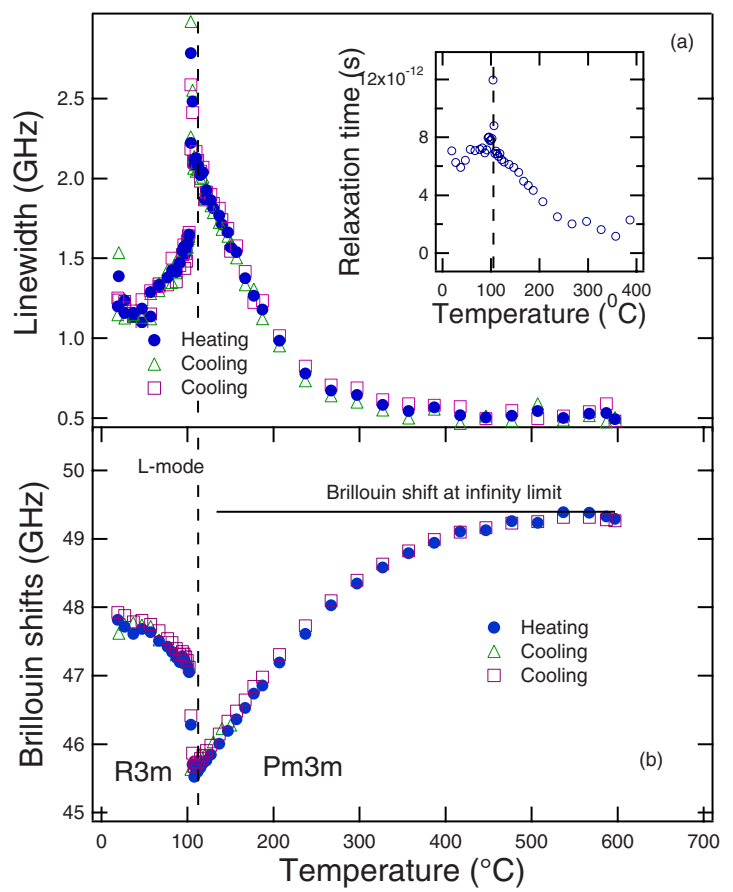

FIG. 2. (Color online) Temperature dependence of quasi-LA mode and its linewidth of $d$-PSN. (a) Solid circles are linewidth values on heating and the open symbols represent the values on cooling. Inset (a) exhibits the relaxation time $\tau_{\mathrm{LA}}(T)$ calculated from Eq. (1) (Ref. 20). (b) Solid circles represent the frequency of the $L$-mode on heating, open symbols on cooling.

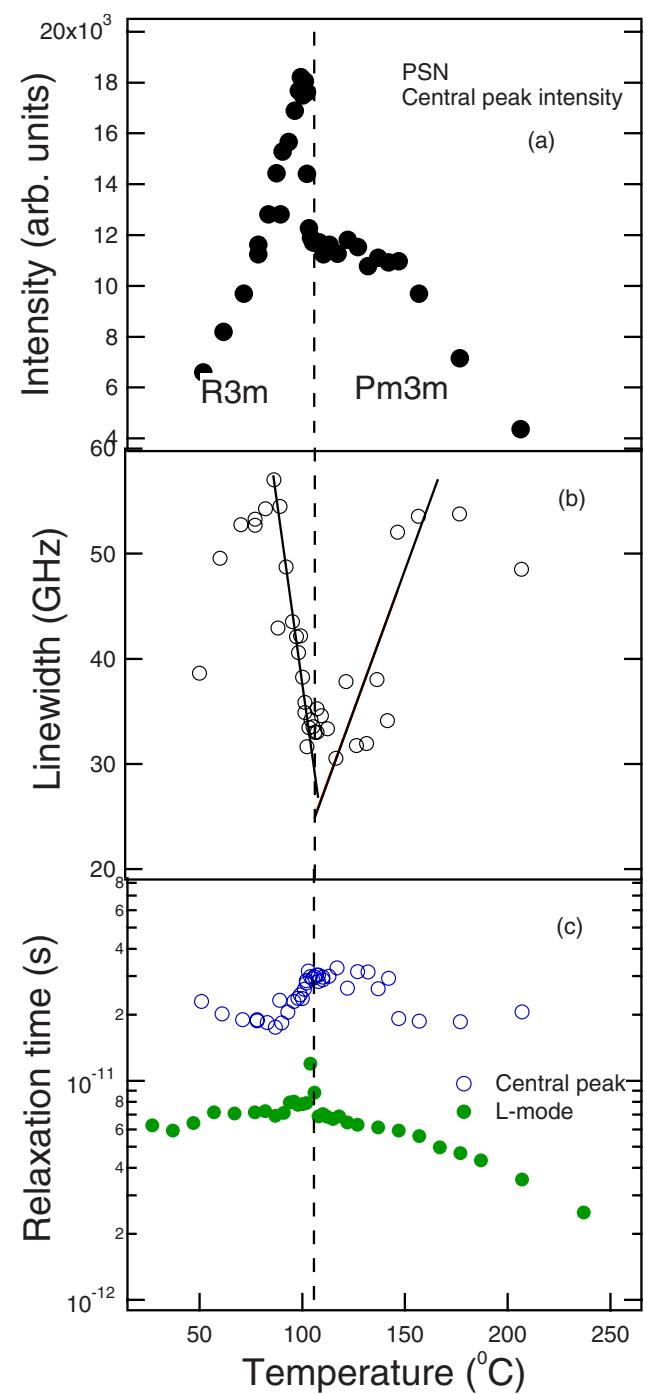

FIG. 3. (Color online) (a) Temperature dependence of the CP intensity of $d$-PSN. (b) Temperature dependence of the CP linewidth; points are experimental data and the lines are fits to Eq. (2). (c) Temperature dependence of the relaxation time $\tau_{\mathrm{CP}}(T)$ determined by the linewidth of $\mathrm{CP}$ and the relaxation time $\tau_{\mathrm{LA}}(T)$ determined by the LA mode.

We observed a $\lambda$-type peak in the temperature dependence of linewidth of LA mode. However, Landau theory cannot explain the significant softening of LA mode between $T_{c}$ and $600{ }^{\circ} \mathrm{C}$. The significant softening of LA mode in cubic phase upon cooling was also observed in PMN-PT systems, ${ }^{13}$ which can be attributed to the coupling between acoustic mode and PNRs below the Burns temperature. ${ }^{1}$ The relaxation time $\tau_{\mathrm{LA}}(T)$ (insets of Figs. 2 and 3) related to LA mode was calculated using the following equation:

$$
\tau_{\mathrm{LA}}(T)=\frac{\Gamma(T)-\Gamma_{\infty}}{2 \pi\left[\nu_{\infty}^{2}-\nu(T)^{2}\right]},
$$

where $\nu_{\infty}$ is the Brillouin shift at infinity limit as shown in Fig. 2(b) by the straight line obtained at the plateau of the Brillouin shifts at higher temperature, $\Gamma(T)$ is the temperature dependent linewidth, $\Gamma_{\infty}$ is the linewidth at the hightemperature limit about $0.5 \mathrm{GHz}$, and the numerator is the difference between the temperature dependent linewidth and the linewidth at the highest temperature. ${ }^{20}$ One of the interpretations of the observed relaxation is that it originates from polarization fluctuations within the PNRs, which in turn couples to the LA mode through density fluctuation. De- 
creasing temperature increases the PNR size, and consequently the interaction between PNRs and the LA mode is enhanced, causing significant softening of the acoustic modes by electrostrictive coupling.

Changes in intensity and two relaxation times occurred near $T_{c}$ in both ferroelectric and nonferroelectric phases are observed (Fig. 3). Both relaxation times revealed here by Brillouin scattering are fast relaxation processes in the gigahertz range; in contrast, dielectric spectroscopy measures slow relaxation $(1 \mathrm{mHz}-\mathrm{MHz})$. The $\mathrm{CP}$ in relaxors can be considered as arising from local polarization fluctuations in PNRs that couple to not only LA but also optical modes. ${ }^{13}$ In contrast with other relaxors, $d$-PSN not only shows relaxor behavior above $T_{c}$, but also exhibits the order-disorder nature of a ferroelectric phase transition. In normal ferroelectrics, the $\mathrm{CP}$ is a characteristic feature of the light scattering spectrum near the phase transition temperature. ${ }^{14}$ The intensity of the $\mathrm{CP}$ can be identified with the intensity of equilibrium fluctuations, and the width of the $\mathrm{CP}$ is inversely proportional to the characteristic relaxation time $\tau$ for the correlation function of these fluctuations. Theoretical models for these systems predict a critical slowing down of the relaxation time near the phase transition temperature $T_{c}$, which corresponds to a decrease in the width of the $\mathrm{CP}$ following ${ }^{14}$

$$
\frac{1}{\tau}=\frac{1}{\tau_{0}} \frac{T-T_{0}}{T_{0}} .
$$

Fits using Eq. (2) in the cubic $\left(T_{c} \sim T_{c}+37{ }^{\circ} \mathrm{C}\right)$ and rhombohedral $\left(T_{c} \sim T_{c}-20{ }^{\circ} \mathrm{C}\right)$ phases yield $T_{0}=60{ }^{\circ} \mathrm{C} \quad\left(\tau_{0}\right.$ $=5.4 \mathrm{ps})$ and $T_{0}=126{ }^{\circ} \mathrm{C}\left(\tau_{0}=31 \mathrm{ps}\right)$, respectively. The incomplete slowing down observed near $T_{c}$ may partly be due to the relaxor nature, the first-order character of the phase transition and the defects included in the crystal. Such behavior has also been observed for many order-disorder ferroelectrics [e.g., $\mathrm{KH}_{2} \mathrm{PO}_{4}$ (Ref. 15)].

The critical slowing down for the width of the CP of $d$-PSN in the nonferroelectric geometry [Fig. 3(b)] and the complex temperature dependence of the relaxation time may be characteristic of ferroelectrics. It should be pointed out that very similar linewidth behavior was observed in lithium tantalate recently. ${ }^{16}$ There may exist different relaxation processes which may be responsible for different behavior of the $\mathrm{CP}$ at different temperatures (e.g., Refs. 17 and 18). Equation (2) predicts that at temperatures far from $T_{c}$, relaxation is dominated by the motion within one unit cell, while close to $T_{c}$ the relaxation response reflects strong collective fluctuations of the order parameter.

Assuming the ferroelectric transition in $d$-PSN is associated to a large extent with the displacement of sublattices, ${ }^{9}$ a specific feature discriminating this transition from an idealized displacive-type transition is the coupling of the softferroelectric mode with the relaxation mode and PNRs. In particular, the PNRs begin to form well above the phase transition in the relaxor phase and these clusters couple to the soft-ferroelectric mode. The coupling between PNRs and the soft-ferroelectric mode observed in PMN by neutron scattering ${ }^{19}$ can be considered as a general feature of $\mathrm{Pb}$ - perovskite relaxors. This coupling may overdamp the soft mode in relaxors and complicate the response. In addition, the coupling between the relaxation and soft-ferroelectric modes gives rise to a transfer of vibrational excitation energy to the relaxation mode when the vibrational frequency is low enough. Accordingly, near $T_{c}$ the collective motion of the soft mode is replaced by the collective relaxation mode. ${ }^{14}$

In conclusion, we have found by Brillouin scattering that PSN undergoes an order-disorder rhombohedral to cubic phase transition at $110{ }^{\circ} \mathrm{C}$. The significant softening of the LA mode upon cooling can be attributed to the electrostrictive coupling of PNRs and an acoustic mode. The temperature dependence of the CP exhibits a critical slowing down in both ferroelectric and nonferroelectric phases near the transition. The behavior of the CP near $T_{c}$ can be understood as an order-disorder-type ferroelectric phase transition. The similarity in temperature dependences of the relaxation times determined from the $\mathrm{CP}$ and LA mode suggests that the former arises from local polarization fluctuations in the PNRs that couple to density fluctuations.

We thank V. Sivasubramanian, S. Tsukada, R. E. Cohen, and S. E. Gramsch for comments. This research was supported by the Grant-in-Aid for Scientific Research (A), 2005, 16204032; the 21st Century COE program under MEXT, Japan; the Office of Naval Research under Grant Nos. N0001402-1-0506 and N00014-06-1-0166; and the Carnegie/ Department of Energy Alliance Center (CDAC, Contract No. DE-FC03-03NA00144).

${ }^{1}$ G. Burns and F. H. Dacol, Solid State Commun. 48, 853 (1983).

${ }^{2}$ S.-E. Park and T. R. Shrout, J. Appl. Phys. 82, 1804 (1997).

${ }^{3}$ V. Bovtun, J. Petzelt, V. Prokhonskyy, S. Kamba, and Y. Yakimenko, J. Eur. Ceram. Soc. 21, 1307 (2001).

${ }^{4}$ J. Hlinka, J. Petzelt, S. Kamba, D. Noujni, and T. Ostapchuk, Phase Transit. 79, 41 (2006).

${ }^{5}$ S. Kamba, M. Kempa, V. Bovtun, J. Petzelt, K. Brinkman, and N. Setter,

J. Phys.: Condens. Matter 17, 3965 (2005).

${ }^{6}$ I. Siny and R. Katiyar, Ferroelectrics 206, 307 (1998).

${ }^{7}$ B. E. Vugmeister and H. Rabitz, Phys. Rev. B 57, 7581 (1998)

${ }^{8}$ C. Perrin, N. Menguy, E. Suard, Ch. Muller, C. Caranoni, and A. Stepanov, J. Phys.: Condens. Matter 12, 7523 (2000).

${ }^{9}$ I. K. Bdikin, V. V. Shvartsman, and A. L. Kholkin, Appl. Phys. Lett. 83, 4232 (2003).

${ }^{10}$ E. L. Venturini, R. K. Grubbs, G. A. Samara, Y. Bing, and Z.-G. Ye, Phys. Rev. B 74, 064108 (2006).

${ }^{11}$ B. P. Burton, E. Cockayne, and U. V. Waghmare, Phys. Rev. B 72, 064113 (2005).

${ }^{12}$ M. Ahart, T. Yagi, and Y. Takagi, Jpn. J. Appl. Phys., Part 1 35, 2882 (1996).

${ }^{13}$ F. M. Jiang and S. Kojima, Phys. Rev. B 62, 8572 (2000).

${ }^{14}$ H. Z. Cummins and A. P. Levanyuk, Light Scattering Near Phase Transitions (North-Holland, Amsterdam, 1983).

${ }^{15}$ M. D. Mermelstein and H. Z. Cummins, Phys. Rev. B 16, 2177 (1977).

${ }^{16}$ A. Hushur, S. Gvasaliya, B. Rossli, S. Lushnikov, and S. Kojima, Phys. Rev. B 76, 064104 (2007).

${ }^{17}$ N. V. Surovtsev, A. M. Pugachev, V. K. Malinovsky, A. P. Shebanin, and S. Kojima, Phys. Rev. B 72, 104303 (2005).

${ }^{18}$ J.-H. Ko, S. Kojima, T.-Y. Koo, J. H. Jung, C. J. Won, and N. J. Hur, Appl. Phys. Lett. 93, 102905 (2008).

${ }^{19}$ P. M. Gehring, S. Wakimoto, Z.-G. Ye, and G. Shirane, Phys. Rev. Lett. 87, 277601 (2001).

${ }^{20}$ S. Tsukada and S. Kojima, Phys. Rev. B 78, 144106 (2008). 